\title{
A Cognitive Autopsy Approach towards Understanding Diagnostic Failure
}

\author{
Pat Croskerry ${ }^{1}$ and Samuel Campbell ${ }^{2}$ \\ ${ }^{1}$ Nova Scotia Health Authority \\ ${ }^{2}$ QEII Health Sciences Centre Foundation
}

November 17, 2020

\begin{abstract}
Diagnostic failure has emerged as one of the most significant threats to patient safety, and it is important to understand the antecedents of such failures. A consensus has developed in the literature that the majority are due to individual or system factors or some combination of the two. A major source of variance in individual clinical performance is due to cognitive and affective biases, however, their role in clinical decision making has been difficult to assess partly because they are difficult to investigate experimentally. A significant drawback has been that experimental manipulations appear to confound assessment of the context surrounding the diagnostic process itself. The present qualitative study uses a detailed narrative account of selected actual cases of diagnostic error to explore the effect of biases in the 'real world' emergency medicine (EM) context. Thirty anonymized EM cases were analysed in depth through a process of root cause analysis that included an assessment of error producing conditions, knowledge-based errors, and how clinicians were thinking and deciding during each case. A prominent feature of the study was the identification of specific cognitive and affective biases - through a process called cognitive autopsy. The cases covered a broad range of diagnoses across a wide variety of disciplines. A total of 24 discrete cognitive and affective biases that contributed to misdiagnosis were identified and their incidence recorded. 5-6 biases were detected per case, and observed on 168 occasions across the 30 cases. Thirteen error-producing conditions (EPCs) were identified. Knowledge-based errors were rare, occurring in only 5 definite instances. The ordinal position in which biases appeared in the diagnostic process was recorded. This study provides a base-line for understanding the critical role that biases play in clinical decision making and sheds light on important aspects of the diagnostic process.
\end{abstract}

\section{Hosted file}

AEM A cognitive autopsy approach towards understanding diagnostic failure_0ct 20 20.pdf available at https://authorea.com/users/376557/articles/493482-a-cognitive-autopsy-approachtowards-understanding-diagnostic-failure 